\title{
Gas inflow and AGN-driven outflow in M51
}

M. Querejeta ${ }^{1}$, S. E. Meidt ${ }^{1}$, E. Schinnerer ${ }^{1}$, S. García-Burillo ${ }^{2}$,

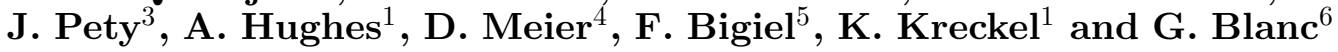

${ }^{1}$ Max-Planck-Institut für Astronomie, Königstuhl 17, D-69117 Heidelberg, Germany

${ }^{2}$ OAN, Madrid, Spain; ${ }^{3}$ IRAM, Grenoble, France; ${ }^{4}$ New Mexico Tech, USA $;{ }^{5}$ University of

Heidelberg, Germany; ${ }^{6}$ Carnegie Institution, USA. Email: querejeta@mpia.de

Abstract. We study the feeding and feedback of the nucleus of M51 by considering gravitational torques, responsible for gas inflow, in relation to the local distribution of dense gas.

Keywords. galaxies: AGN — galaxies: structure — galaxies: evolution

\section{Context}

Feedback from AGN is often invoked as a mechanism that regulates star formation. In spite of its cosmological relevance, the way it operates in practice is poorly understood, apart from the basic idea that it must somehow remove the fuel of star formation (cold dense gas) from the disc. The low inclination $\left(\sim 22^{\circ}\right)$ and proximity of M51 (7.6 Mpc) make it an ideal target to directly link the feeding of the AGN with its feedback.

\section{Torques driving gas inwards}

Gravitational torques add or remove angular momentum from the gas $(\tau=d L / d t)$, thus effectively driving it outwards or inwards. We use our ICA-corrected $3.6 \mu \mathrm{m}$ mass map (Querejeta et al. 2014) and CO(1-0) imaging from PAWS (Pety et al. 2013) to measure the gravitational torques in M51. These imply clear gas inflow to fuel nuclear activity over the entire central region, at an integrated rate of $\sim 20 M_{\odot} / y r$ inside 20 ".

\section{Molecular outflow}

A redshifted and possible blueshifted peak in the CO PAWS velocity field are likely signatures of a molecular outflow. We have obtained new Plateau de Bure (PdBI) observations of the nucleus in three dense gas tracers: $\mathrm{HCN}, \mathrm{HCO}^{+}$and $\mathrm{HNC}$. An off-centered, lopsided dense gas feature along the radio jet is clearly visible in the morphology and kinematics. Interestingly, $\mathrm{HCN}$ shows more extreme velocities than $\mathrm{CO}$ in this region, suggesting a high degree of jet-ISM interaction.
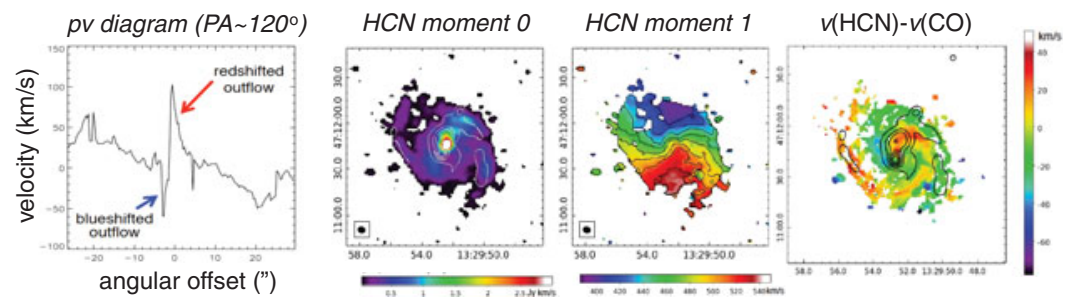

Figure 1. Position-velocity diagram in the center of the PAWS CO map (PA $120^{\circ}$ ), pointing to the outflow. HCN maps exhibit a significant velocity difference with respect to CO. 